\title{
Cambio de paradigma en la educación
}

\author{
Paradigm shift in education \\ Rosa Ma. Guzmán Aguilar, ${ }^{*}$ José Arturo Vázquez, ${ }^{\ddagger}$ Abilene Escamilla Ortiz ${ }^{\S}$
}

\author{
Palabras clave: \\ Educación a \\ distancia, simulación, \\ tecnologías de \\ la información y \\ comunicación, \\ aprendizaje, sociedad \\ de la información, \\ e-learning en \\ educación médica. \\ Keywords: \\ Distance education, \\ simulation, \\ information and \\ communications \\ technology, learning, \\ information society, \\ e-learning in medical \\ education.
}

${ }^{*}$ Coordinadora de Academia Virtual, Asociación Mexicana de Cirugía General, A.C. Ciudad de México, México.

‡ Coordinador de la Carrera de Medicina de la Universidad IEST-Anáhuac. Tampico, Tamaulipas. $\S$ Editora, revista Cirujano General. Ciudad de México, México.

Recibido: 15/06/2020 Aceptado 07/07/2020

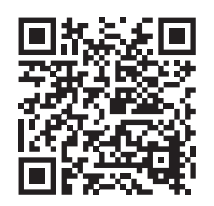

\section{RESUMEN}

El aprendizaje durante la pandemia ha dado un giro de un día para otro, los cambios tecnológicos acelerados han generado una "nueva normalidad" y han hecho que se tenga que utilizar de manera eficiente las tecnologías de la información y comunicación, lo mismo que la simulación en educación médica y quirúrgica. La evolución de los espacios virtuales no es sencilla, acarrea adopción de nuevos procesos de enseñanza-aprendizaje. La educación a distancia es una oportunidad, logrando así no suspender actividades académicas y optimizar la disponibilidad del tiempo. La simulación clínica y quirúrgica, la cual puede ayudar a sustituir las prácticas, se lleva a cabo sobre escenarios planeados y estructurados de forma similar a la realidad. Permite evaluar al alumno a través de una intervención con él para hacerle saber sus errores y aciertos, todo esto mediante el análisis del uso de rúbricas. Las redes sociales se pueden utilizar para la educación, se pueden ver y escuchar conferencias virtuales y congresos. Para continuar la enseñanza con los residentes, se pasó del aula a las sesiones virtuales, para complemento se puede tener un repositorio de videos, grupos cerrados de Facebook ${ }^{\odot}$ donde se puede interactuar con los residentes a través de preguntas, cuestionarios o poner temas a debate. La tendencia mundial es incrementar la carga académica fuera del aula tradicional, es decir, en espacios virtuales que vinculen la simulación y exploten al máximo las tecnologías de la información y comunicación.

\section{ABSTRACT}

Learning during the pandemic has taken a turn from one day to the next, accelerated technological changes have generated a "new normality", they have made it necessary to use information and communication technologies efficiently as well as simulation in medical and surgical education. The evolution of virtual spaces is not simple, it entails the adoption of new teaching-learning processes. Distance education is an opportunity, thus managing not to suspend academic activities and optimize the availability of time. The clinical and surgical simulation, which can help to replace the practices, is carried out on planned and structured scenarios similar to reality. It allows evaluating the student through an intervention with him to let him know his mistakes and successes, all this through the analysis of the use of rubrics. Social networks can be used for education, you can see and listen to virtual conferences, congresses. To continue teaching with the residents, the classroom was moved to the virtual sessions, to complement it, you can have a video repository, closed Facebook ${ }^{\odot}$ groups where you can interact with the residents through questions, questionnaires, or put topics for debate. The global trend is to increase the academic load outside the traditional classroom, that is, in virtual spaces that link simulation, and make the most of information and communication technologies.

\section{INTRODUCCIÓN}

$\mathrm{E}^{\prime}$ objetivo de este documento es discutir sobre el posicionamiento apremiante del aprendizaje en espacios virtuales a partir de una sociedad del conocimiento abrumada por cambios tecnológicos acelerados y eventos epidemiológicos que han generado una "nueva normalidad" asociada con abruptos movimientos económicos, tecnocientíficos, sociales y políticos, entre otros, que apremian la utilización eficiente de las tecnologías de la información y comunicación así como de la simulación en educación médica y quirúrgica.

\section{El cambio como primicia en el ámbito educativo}

Nunca como hoy en día en este mundo globalizado la necesidad del cambio ha sido tan

Citar como: Guzmán ARM, Vázquez JA, Escamilla OA. Cambio de paradigma en la educación. Cir Gen. 2020; 42(2): 132-137. doi: 10.35366/95373 
forzosa para dar continuidad a los procesos. Los fenómenos económicos, políticos y sociales de nuestro tiempo han transformado radicalmente el entorno, imponen nuevos retos, mayor demanda de capital humano y exigen trabajo colaborativo de calidad. Frente a este panorama, la educación no escapa a esta realidad y actúa en consecuencia con la implementación de nuevos paradigmas educativos, con lo cual no sólo estamos propiciando un mejor futuro, más importante aún, estamos transformando el presente.

La globalización y el vertiginoso desarrollo de las tecnologías de la información y comunicación (TIC's) han generado una dependencia de la inmediatez y con ello de las herramientas tecnológicas. En la actualidad ninguno de nosotros nos imaginamos sin el uso de la telefonía celular que nos permita contactar a individuos que se encuentran en locaciones distantes y que a la vez este mismo celular nos informe las noticias relevantes del día, el estado del clima, los cambios en la bolsa de valores o nos haga partícipes en sólo minutos de comunidades virtuales a través de las redes sociales como Facebook, Twitter, WhatsApp, etc. Deambulamos en medio del conocimiento, la cultura y la mercantilización del ocio, donde la tecnología los entremezcla y explota en multiplicidad de modalidades, a través de las cuales las redes sociales, videos, televisión o videojuegos contribuyen en la enseñanza, estimulan la resolución de problemas educativos y a su vez, al esparcimiento.

Las necesidades sociales y económicas demandan cambios radicales en la forma de enfrentar los retos, tomar decisiones y solucionar problemas, por ende la educación ha dado un giro donde posiciona al estudiante como el protagonista principal del proceso de enseñanza-aprendizaje, gestor de la construcción de sus conocimientos, autónomo y responsable de satisfacer sus necesidades de formación, motivado para aterrizar su proyecto de vida como un individuo feliz en constante perfeccionamiento para transformar su entorno en ambientes armónicos que le permitan alcanzar sus metas y el bienestar personal y colectivo.

Bajo esta perspectiva, la educación en línea surge como réplica a las demandas de la glo- balización, la tecnocracia y una sociedad del conocimiento apremiantes de capital humano con pensamiento sistémico asociado a competencias cognitivas, habilidades y actitudes positivas que den respuesta a los requerimientos del sector laboral.

No obstante lo anterior, en México el paradigma educativo en espacios virtuales y sus actores (profesores, alumnos, instituciones educativas, comunidad, etc.) se encuentra sorteando la curva de aprendizaje con diferentes grados de madurez, con avances escalonados y no uniformes entre los diferentes estratos socioeconómicos al interior del país.

\section{Evolución y pertinencia en la educación a distancia (EaD)}

La guerra y las pandemias comparten la necesidad de fortalecer la fuerza laboral y la innovación mediante la construcción de nuevos conocimientos y formas de empleabilidad para evitar la recesión económica. Mediante este precepto, las innovaciones en tecnologías para la educación en espacios virtuales apuntan hacia una revolución educativa que exige pertinencia en la estructuración de contenidos con base en escenarios contextualizados, necesidades emergentes del cambio y sobre todo, que propicien visualizar y prever los eventos del futuro.

El proceso evolutivo de los espacios virtuales no es sencillo, ya que acarrea adopción tanto de diversos patrones de conducta como del propio proceso de enseñanza-aprendizaje, ya que el cruce de dicho espacio da pie a sumergirse en zonas de posibles malentendidos entre los aportes y percepciones del instructor y del alumno, en virtud de que los espacios psicológicos y de comunicación entre estudiante e instructor nunca son exactamente los mismos, ya que aún en educación presencial hay cierta distancia transaccional que requiere ser acotada, ${ }_{1}^{1}$ por lo tanto las estrategias y técnicas específicas para el aprendizaje en $\mathrm{EaD}$ muestran conceptos psicopedagógicos e instruccionales distintivos que transforman el papel de profesor en facilitador y más aún en catalizador de los requerimientos individuales del alumno, así como para estimular las interacciones entre los miembros del equipo de aprendizaje. 
Aunque la masificación de la EaD en el ámbito quirúrgico se enfoca en resolver la creciente demanda de programas continuos de capacitación o actualización así como el aprovechamiento de los vertiginosos avances tecnológicos del siglo XXI, implica un gran desafío, ya que requiere contar con la infraestructura adecuada, personal calificado en Educación y Tecnologías de la Información y Comunicación, así como mantenimiento continuo de la plataforma e incorporación de nuevos objetos de aprendizaje.

Los elementos de mayor impacto en la EaD son el diseño del programa, la explotación de diversos medios y recursos, así como estilos de comunicación, características emocionales, tipo de personalidad y estilos de aprendizaje de los alumnos que promuevan creatividad, autorregulación, compromiso y responsabilidad del binomio.

\section{La "nueva normalidad" como oportunidad para adoptar un cambio en la educación}

La conmoción originada por el COVID-19 en el mundo, y sin lugar a dudas en el sector educativo, abre una gran oportunidad para adoptar a nivel global el cambio en la EaD más grande de la historia y de esta manera abordar a nuestro favor las circunstancias de confinamiento así como reducir la suspensión de las actividades académicas y optimizar la disponibilidad del tiempo. $^{2}$

Esta inesperada y abrupta realidad embebida en una multiplicidad de percepciones del entorno nos sumerge en una danza de emociones, mismas que transitan entre el miedo y la incertidumbre por la perpetuación de los métodos tradicionales de transmisión de información y reproducción de constructos obsoletos que impera en nuestro sistema educativo desde el nivel básico hasta el posgrado. La "nueva normalidad" invita a dar sentido a lo prioritario, emergente, novedoso y eficaz para convertirnos en observadores diferentes de la realidad, abiertos al cambio y desafiantes para sacar a flote competencias latentes y hacer sinergia de talentos que propicien el trabajo colaborativo para la conversión de las amenazas en oportunidades.

Esta "nueva normalidad" convergente con la EaD rompe las barreras del tiempo y espacio, acerca a la población en lo individual y en lo colectivo a una gamificación de contacto audiovisual que vence sin lugar a dudas el aislamiento; ${ }^{3}$ posiciona al sujeto como protagonista, responsable de su aprendizaje y promotor de conversaciones que germinen escenarios mentales armónicos y amigables para la interacción e intercambio de experiencias que incentiven atmósferas de pertenencia y logro para una toma de decisiones asertivas que abran nuevas formas de enfrentar la complejidad de la situación actual.

En el presente siglo la educación médica está viviendo cambios interesantes y determinantes asociados con los avances en el conocimiento y la tecnología; la evolución epidemiológica y las exigencias de los pacientes han llevado a las instituciones a reformular su manera de enseñar la medicina. ${ }^{1}$

\section{Simulación estrategia educativa en tiempos de COVID-19}

En estos momentos de crisis educativa condicionada por la pandemia del virus COVID-19 se han buscado recursos virtuales para cubrir la falta de presencialidad de los estudiantes en la universidad; además de esto, surge la preocupación de cómo atender la práctica clínica. ${ }^{4}$

Aunque la EaD representa una herramienta relevante en el aprendizaje y evaluación de conceptos teóricos y habilidades como la comunicación, liderazgo y toma de decisiones, entre otras; la formación de los nuevos cirujanos enfrenta retos importantes en la adquisición de competencias en torno a la práctica clínica y destrezas quirúrgicas, donde la simulación clínica es un importante elemento para la enseñanza. ${ }^{5}$

La simulación clínica es una disciplina que se define como un conjunto de métodos que facilitan a los médicos en formación la adquisición de habilidades y destrezas en escenarios similares a la vida real en un ambiente seguro de aprendizaje con la finalidad de no poner en riesgo a los pacientes. Actualmente esta disciplina representa una de las estrategias de enseñanza que permite a un estudiante de medicina o residente de cualquier especialidad, frente a escenarios planeados, diseñados y estructurados de una forma similar a la realidad, 
fortalecer el desarrollo de habilidades destrezas, actitudes y aptitudes teniendo como objetivo mantener la humanización de la práctica médica, y por encima de todo la seguridad de los pacientes fomentando el trabajo en equipo.

Es importante señalar que la implementación de la simulación clínica no da la espalda a los procesos de enseñanza presenciales en la educación médica y quirúrgica, ni sustituye la práctica con el paciente real, sino todo lo contrario; éste representa un complemento y un enlace entre la fase de ciencias básicas y la clínica, actúa como un puente entre lo teórico y lo práctico, con la ventaja de poder repetir las veces que sea necesario un procedimiento hasta dominarlo y después realizarlo de manera segura con menor grado de complicaciones en el paciente real; acorta sin duda la curva de aprendizaje y mediante el debriefing (conversación entre dos o más personas para revisar el evento simulado), si el alumno explora y analiza sus acciones, descubre el error, reflexiona sobre sus estados emocionales y una nueva información para mejorar su rendimiento en situaciones reales. ${ }^{6,7}$

En estos tiempos de confinamiento no debemos caer en el error de pensar que la simulación puede sustituir en su totalidad la práctica quirúrgica con pacientes en escenarios reales; sin embargo, los centros de simulación propician el desarrollo de destrezas y habilidades de manera eficiente, dado que cuentan con espacios controlados y contextualizados que permiten la presencialidad del alumno y adoptan temáticas centradas en el "hacer" y en el mostrar "cómo lo hace" con base en las evidencias de aptitud superior establecidas en la pirámide de Miller del conocimiento, por lo que constituyen una solución eficiente al impedimento de presencialidad del estudiante y para la práctica clínica en tiempos actuales de pandemia.

En virtud de lo anterior, surgen cuestionamientos sobre el campo de acción y contribución de la simulación clínica para el desarrollo de competencias del cirujano en tiempos de pandemia. Al respecto, la simulación de baja fidelidad requiere de partes anatómicas o de simuladores de tareas específicas para nudos y suturas, ejercicios de laparoscopia o anastomosis, entre otros, que pudieran requerir la presencia del alumno y en la actualidad se pueden implementar a la distancia, si se tiene a la mano el recurso, por ejemplo, se puede grabar al alumno o residente haciendo su caja de entrenamiento, y el profesor puede ver lo realizado por el alumno y hacer las recomendaciones pertinentes.

Por otro lado, existen dinámicas como las sesiones en video, donde a partir de casos clínicos simulados o de eventos quirúrgicos se puede interactuar a distancia con la finalidad de desarrollar el razonamiento clínico o habilidades como la toma de decisiones; esto se conoce en algunos medios como telesimulación. ${ }^{6}$

Finalmente concluimos, aunque aún no termina esta inesperada batalla que el personal de salud enfrenta contra el COVID-19, que las lecciones aprendidas nos inducen a evaluar críticamente los métodos actuales de entrega educativa frente a este panorama incierto $y$ cambiante, ${ }^{8}$ por lo que es imperativo vivir el ahora con una visión empoderada, ávida de superación y emprendedora para desarrollar estrategias de $\mathrm{EaD}$ y simulación que minimicen las interrupciones de los programas presenciales de capacitación en el personal médico en formación y de actualización permanente en todas las especialidades quirúrgicas.

La tendencia mundial es incrementar la carga académica fuera del aula tradicional, es decir, en espacios virtuales que vinculen la simulación y exploten al máximo las tecnologías de la información y comunicación para construir experiencias de aprendizaje al ritmo particular del alumno y más aún, que le permitan identificar los puntos más relevantes y coyunturales para descubrir o crear oportunidades para el progreso y cimentación de nuevos conocimientos.

\section{Uso de redes sociales en la educación quirúrgica}

Durante esta pandemia el uso de las redes sociales como Facebook, Twitter, YouTube, Instagram, Snapchat, entre otras, son la fuente más importante para expandir las noticias sobre COVID, lamentablemente no todas las noticias son reales, por el contrario, son falsas, hay mucha desinformación. ${ }^{9}$ Casi dos millones de personas en el mundo hacen uso de las 
redes sociales durante la pandemia, lo que ha ocasionado que estemos más tiempo frente a la pantalla, se ha cambiado la venta tradicional por la venta en línea, y se ha tenido que llevar la enseñanza a través de esta modalidad. ${ }^{10}$ De igual forma los negocios, si las utilizan de una manera inteligente y creativa, pueden tener resultados positivos. Han surgido plataformas nuevas como TikTok y EduTok que es la parte educativa. También el uso de Podcast y Telegram están cambiando el escenario en las redes sociales. ${ }^{10}$

Derivado de la pandemia se ha observado que el uso de las redes sociales ha sido útil, sobre todo para proporcionar información médica que se considera verdadera, pero también se ha caído en la infodemia en la que prevalecen las notas falsas, sobre todo porque se usa la imagen de una enfermera o un médico para dar estas notas; es por eso que plataformas como Facebook, por ejemplo, direccionan al usuario a los sitios de la Organización Mundial de la Salud para información confiable. Las redes sociales se pueden utilizar para la educación. A través de ellas es posible ver y escuchar conferencias virtuales sobre todo para aprender en cuanto al COVID-19 y generar discusión, esto debido a que varios congresos o conferencias se han cancelado por la pandemia. ${ }^{11}$ ¿Qué debemos hacer para evitar la infodemia? Se debe seguir a autoridades sanitarias y a especialistas reconocidos y confiables en la información que proporcionan. Diario llega información a nuestras redes, correos, WhatsApp, por lo tanto no debemos circular información que no ha sido verificada. ${ }^{11}$

\section{¿Cómo podemos usar la tecnología con los residentes durante esta pandemia?}

Desde que dio inicio la pandemia, tanto el Colegio Americano como Cirujanos y la Asociación Mexicana de Cirugía General determinaron suspender la cirugía programada, únicamente llevar a cabo la cirugía urgente, no juntar a más de 10 personas, y disminuir el personal en la sala de operaciones; esto hizo cambiar de un día para otro la enseñanza quirúrgica en los residentes. Se han hecho algunas propuestas para continuar con ésta misma haciendo uso de la tecnología. ${ }^{12}$
Al transferir el trabajo del aula fuera de ésta, hay que dar adecuadamente las instrucciones de lo que se quiere y fomentar la participación de los alumnos con preguntas y discusiones. Se puede tener un repositorio de videos que el residente puede ver después, los videos deben ser diferentes de acuerdo con la jerarquía. Otra opción que se planteó fue abrir un grupo cerrado en Facebook donde se suben preguntas diarias con el fin de prepararlos para su examen de certificación y de invitarlos a las conferencias académicas de nuestra asociación o de otras asociaciones relacionadas con la cirugía. Para las clases hay diferentes plataformas gratuitas que se pueden utilizar desde el celular, la tableta o la computadora, se recomienda grabar las sesiones para que se queden en la nube y estén a la disposición de los residentes. ${ }^{12}$

En Twitter hay diferentes cuentas de educación quirúrgica y de cirujanos que suben sus casos clínicos y se produce una interacción mediante la opinión; también se suben temas y artículos para revisión y se genera buena discusión siempre tratando de que sea medicina y cirugía basada en evidencia. Subir los casos debe ser, como ya lo hemos descrito, con el consentimiento del paciente, tiempos, etcétera.

A partir de ahora debemos considerar todas las herramientas que tenemos a la mano para innovar en la educación quirúrgica.

\section{REFERENCIAS}

1. Moore M. Theory of transactional distance. In: Keegan D, ed. Theoretical principles of distance education. Routledge; 1997. pp. 22-38. [Consultado 22 de mayo de 2020] Disponible en: http://www.c3l.unioldenburg.de/cde/found/moore93.pdf

2. Yan Z. Unprecedented pandemic, unprecedented shift, and unprecedented opportunity. Hum Behav Emerg Technol [Internet]. 2020 [Consultado el 26 de mayo de 2020]. Disponible en: https://www.ncbi.nlm. nih.gov/pmc/articles/PMC7228313/

3. Osipov IV, Volinsky AA, Nikulchev E, Prasikova AY. Study of gamification effectiveness in online e-learning systems. IJACSA [Internet]. 2015 [Consultado el 26 de mayo de 2020]; 6: 71-77. Disponible en: https:// thesai.org/Downloads/Volume6No2/Paper_11Study of_Gamification_Effectiveness_in_Online_e-Learning Systems.pdf

4. Díaz-Guio DA, Ferrero F, Vázquez-Vázquez JA, et al. Educación en línea: un camino hacia la formación docente sin fronteras. Simulación Clínica. 2019; 1 : 121-122. 
5. Cabero-Almenara J. Bases pedagógicas del e-learning RUSC [Internet]. 2006 [Consultado el 14 de junio de 2020]; 3. Disponible en: https://www.redalyc.org/ pdf/780/78030102.pdf

6. Ruiz-Parra AI, Angel-Müller E, Guevara O. La simulación clínica y el aprendizaje virtual. Tecnologías complementarias para la educación médica. Rev Fac Med. 2009; 57: 67-79.

7. Amaya A. El razonamiento clínico un objetivo de la educación médica. Universitas Médica. 2008; 49: 289-292.

8. Plancher KD, Shanmugam JP, Petterson SC. The changing face of orthopedic education: searching for the new reality after COVID-19. Arthrosc Sports Med Rehabil [Internet]. 2020 [Consultado el 26 de mayo de 2020]. Disponible en: https://www.ncbi.nlm.nih. gov/pmc/articles/PMC7183965/pdf/main.pdf

9. Hussain W. Role of social media in COVID-19 pandemic. Int J Front Sci. 2020; 4. doi: 10.37978/ tijfs.v4i2.144.

10. Ghosh R. Psychological impact of social media during COVID-19 pandemic lockdown. Int J Mult Edu Research. 2020 5: 171.

11. Laurentino Lima D, Albanez A de Medeiros Lopes MA, Brito AM. Social media: friend or foe in the COVID-19 pandemic? Clinics. 2020; 75: e1953.
12. Chick RC, Clifton GT, Peace KM, Propper BW, Hale DF, Alseidi AA, et al. Using technology to maintain the education of residents during the COVID-19 pandemic. J Surg Educ. 2020; 77: 729-732.

Consideraciones y responsabilidad ética: Privacidad de los datos. De acuerdo a los protocolos establecidos en el centro de trabajo de los autores, éstos declaran que han seguido los protocolos sobre la privacidad de datos de pacientes preservando su anonimato. El consentimiento informado del paciente referido en el artículo se encuentra en poder del autor.

Financiamiento: No se recibió apoyo financiero para la realización de este trabajo.

Conflicto de intereses: Los autores declaran que no existe ningún conflicto de intereses en la realización del trabajo.

\author{
Correspondencia: \\ Dra. Rosa Ma. Guzmán Aguilar \\ E-mail: dra.guzman.aguilar@gmail.com
}

